Emilio Andrea Maugeri*, Jörg Neuhausen, Borja Gonzalez Prieto, Alexander Aerts, Tania Melo Mendonça, Thierry Stora and Robert Eichler

\title{
Adsorption of volatile polonium species on metals in various gas atmospheres: Part III - Adsorption of volatile polonium on stainless steel $316 \mathrm{~L}$
}

https://doi.org/10.1515/ract-2017-2807

Received April 20, 2017; accepted July 10, 2017; published online August 18, 2017

\begin{abstract}
This article summarizes the results obtained studying the interaction between polonium and stainless steel 316L in different atmospheres by the thermochromatography method. This issue is particularly important in terms of licensing of the MYRRHA prototype ADS reactor, where highly radiotoxic $\alpha$-emitting polonium isotopes are produced in lead-bismuth eutectic used as both spallation target material and coolant, while stainless steel 316L is going to be the main structural material. The polonium adsorption enthalpy on stainless steel 316L in inert atmosphere was measured as $-147 \pm 6 \mathrm{~kJ} \mathrm{~mol}^{-1}$. The findings obtained using reducing atmospheres show that the affinity of polonium for stainless steel 316L increases with the metallic character of the stainless steel surface. The presence of moisture in inert and reducing atmosphere does not significantly influence the polonium adsorption. Oxidizing atmospheres induce the formation of polonium dioxide which has a stronger interaction with the stainless steel 316L, with an adsorption enthalpy of $-225 \pm 6 \mathrm{~kJ} \mathrm{~mol}^{-1}$. A prolonged exposure of the stainless steel to strong oxidants, such as pure oxygen, reduces the metallic character of the stainless steel surface and, consequently, its affinity to the polonium oxide. Furthermore, it was found that in all experiments at least $5 \mathrm{ppm}$ of the total polonium present in the starting material were not retained on the stainless steel 316 Lurface at all.
\end{abstract}

*Corresponding author: Emilio Andrea Maugeri, Laboratory for Radiochemistry, Paul Scherrer Institut, Villigen PSI, CH-5232 Villigen, Switzerland, E-mail: emilio-andrea.maugeri@psi.ch Jörg Neuhausen: Laboratory for Radiochemistry, Paul Scherrer Institut, Villigen PSI, CH-5232 Villigen, Switzerland Borja Gonzalez Prieto and Alexander Aerts: SCK · CEN, Chemistry and Conditioning Programme, Belgian Nuclear Research Centre, Boeretang 200, B-2400 Mol, Belgium

Tania Melo Mendonça and Thierry Stora: ISOLDE, CERN, CH-1211 Geneva, Switzerland

Robert Eichler: Laboratory for Radiochemistry, Paul Scherrer Institut, Villigen PSI, CH-5232 Villigen, Switzerland; and Department for Chemistry and Biochemistry, University of Bern, CH-3012 Bern, Switzerland
Keywords: Polonium, thermochromatography, accelerator driven system (ADS), MYRRHA, stainless steel 316L.

\section{Introduction}

Accelerator driven systems (ADS) [1] have been proposed to transmute the long-lived minor actinides into radionuclides with shorter half-life [2]. Liquid lead-bismuth eutectic (LBE) was selected as target material and coolant for MYRRHA [3], the first ADS prototype. In the safety assessments of MYRRHA the volatilization of the isotopes of polonium, ${ }^{208-210} \mathrm{Po}$, and their interaction with construction materials play an essential role. Polonium isotopes are formed in both coolant and spallation material by the interaction of high energetic protons and neutrons with bismuth. Those isotopes are extremely hazardous because of their high radiotoxicity, as they are $\alpha$-emitters with relatively short half-lives. In addition, in particular conditions, polonium tends to form chemical species with high volatility, making this element even more harmful. Maugeri et al. [4] reported the formation of more than one polonium species which are more volatile than the elemental polonium when moisture is present in inert and reducing atmosphere. Thus, the formation of volatile polonium species, induced by reactions with water, represents one of the main safety issues of an $\operatorname{ADS}[5,6]$. Several methods have been considered for removing polonium directly from the LBE, see e. g. Refs. $[5,7,8]$, or to permanently absorb it from the gas plenum above the LBE using dedicated filter materials. For the latter purpose, the first two papers of this series studied the interaction of polonium with different noble metals as candidates for filtering materials $[9,10]$.

This work describes a systematic study on the interaction between polonium in the gas phase and stainless steel (SS316L), used as construction material in MYRRHA, in different atmospheres resembling ADS normal operation, maintenance, and accidental scenarios. To assess the transport of polonium vapor molecules in the reactor cover gas space and in the containment building, it is therefore 
important to have a good knowledge on the interaction of elemental polonium or polonium containing species with stainless steel, which is still lacking. According to Obara et al. [11-13], who studied the interaction between polonium and stainless steel in the view of using this material as filter, between $88 \%$ and $97 \%$ of polonium could be removed from the gas phase by using a stainless steel wire mesh at temperature between $503 \mathrm{~K}$ and $878 \mathrm{~K}$, although neither information about polonium chemical speciation nor thermodynamic parameters needed for an appropriate safety assessment were given.

The present investigation was carried out using the thermochromatography method (TC) [14, 15]. A detailed description of the method used is given in the first papers of this series $[9,10]$.

\section{Experimental}

\subsection{Sample preparation}

The investigation was carried out using trace amounts of carrier free ${ }^{206} \mathrm{Po}$ to avoid formation of diatomic species such as $\mathrm{Po}_{2}$ and $\mathrm{BiPo}$ during the thermochromatography experiment. The isotope ${ }^{206} \mathrm{Po}$ was chosen since it has easily detectable $\gamma$-ray emissions and a suitable half-life of 8.8 days. Carrier free ${ }^{206} \mathrm{Po}$ was produced at the CERNISOLDE facility, where its precursor ${ }^{210} \mathrm{Fr}$ was implanted in thin $(20 \mu \mathrm{m})$ gold foils (Goodfellow, $99.95 \%$ purity) of $8 \mathrm{~mm} \times 4 \mathrm{~mm}$ size in the same manner as described in Refs. $[4,16]$.

\subsection{Thermochromatography}

The TC setup used in this work consisted of a SS316L column $\left(d_{\mathrm{i}}=4.0 \mathrm{~mm}, l=120 \mathrm{~cm}\right),(1)$ in Figure 1, encapsulated in

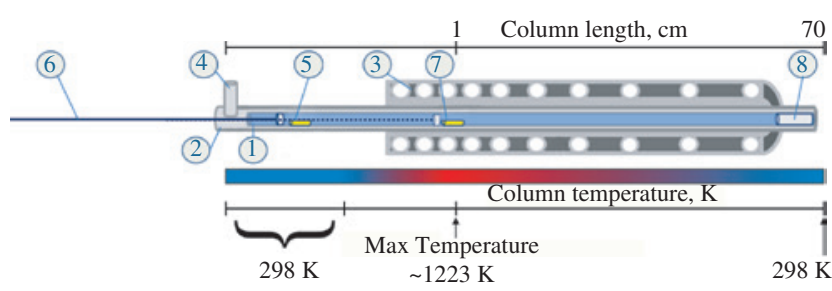

Figure 1: Thermochromatography set-up: 1 - SS316L column; 2 - Incone $\mathrm{l}^{\circledR}$ tube; 3 - negative gradient furnace; 4 - carrier gas inlet; 5 - sample in the starting position (room temperature); 6 - sample introduction system; 7 - sample at position corresponding to the max temperature (1223 K); 8-2 cm silver tube. an Inconel ${ }^{\circledR}$ tube (2) partially placed in a negative temperature gradient furnace (3), with a temperature profile ranging from $1223 \mathrm{~K}$ (high enough to let the investigated species desorb quickly from the starting material) to $298 \mathrm{~K}$. The inlet of the Inconel ${ }^{\circledR}$ tube was connected, via Swagelok ${ }^{\circledR}$ components, to a gas supply (4) allowing for the use of different atmospheres, i.e. inert, reducing, oxidizing. The gas flow rate, controlled by a mass flow controller (Brooks, $5850 \mathrm{~S}$ series), was $29 \pm 0.5 \mathrm{~mL} \mathrm{~min}^{-1}$. For experiments in dry atmospheres helium and hydrogen were passed through both a SICAPENT ${ }^{\circledR}$ cartridge and a tantalum getter heated to $1273 \mathrm{~K}$, to remove traces of water and oxygen. Oxygen was passed only through a SICAPENT ${ }^{\circledR}$ cartridge to remove water traces. When moist carrier gas was used, the gas was moisturized by passing it through a demineralized water reservoir kept at room temperature located before the gas inlet. The moisture content in the gas phase in the experiments performed with water saturated carrier gas was not monitored in each individual experiment, but determined to be in the order of 2 vol- $\%$ in a series of preceding experiments performed under the same experimental conditions using a Michell Easidew ${ }^{\circledR}$ dew-point transmitter. The amount of residual water in the dried carrier gases was measured in each individual experiment using a Michell Pura OEM ${ }^{\circledR}$ trace moisture transmitter placed at the outlet of the gradient furnace. Experiments were performed both in nonpretreated SS316L columns as obtained from the supplier and in columns pretreated for $90 \mathrm{~min}$ at $973 \mathrm{~K}$ in a hydrogen stream of $50 \mathrm{~mL} / \mathrm{min}$ to study the potential influence of reduction on the adsorption behavior.

The starting material was loaded in a sample holder made of stainless steel and placed in the starting position (5) i. e. in a part of the column not inserted into the gradient, where the temperature was about $298 \mathrm{~K}$. Then, the carrier gas flow and the gradient furnace were switched on, and the system was heated until a stable gradient was achieved. This usually took 210-300 min. During this time, the evolution of the gradient was monitored by a K-type thermocouple inserted through the outlet of the column. The temperature profile along the column was then measured in steps of $1 \mathrm{~cm}$. In some experiments, when using reactive carrier gas, the columns were kept in the gradient furnace for longer time, up to a maximum of $1020 \mathrm{~min}$, to study the modifications induced by the carrier gas on the SS316L column. Those modifications, in fact, may influence the interactions between polonium and the stationary phase. In the latter experiments, during the prolonged exposure to the gradient the system was connected to an exhaust system consisting of a $7 \mathrm{~m}$ long perfluoralkoxy polymer tubing ending in a gas bubbler filled with 
silicone oil. The setup was only opened for approximately $30 \mathrm{~min}$ before the experiments to measure the temperature gradient.

After measurement of the gradient, the sample was pushed to the position of the SS316L column corresponding to the maximum temperature, i.e. $1223 \mathrm{~K}$ (7), using a sealed piston pushing system (6) to minimize the ingress of air into the setup. This was considered as time zero of the experiment. A silver tube of $2 \mathrm{~cm}$ length (Goodfellow, thickness $0.020 \pm 0.003 \mathrm{~mm}$, purity $99.9 \%$ ) was lining the inside of the end of each column (8), where the temperature was approximately $298 \mathrm{~K}$, to trap polonium species not retained on the SS316L surface. The activity of ${ }^{206} \mathrm{Po}$ was measured by a HPGe- $\gamma$ detector in comparison with measurements, in the same geometry, of certified standard reference sources. All the experiments were run for $120 \mathrm{~min}$. Afterwards, the gradient furnace was switched off and allowed to cool down to room temperature. Then, the SS316L column was removed from the thermochromatography set-up and investigated by measuring the distribution of ${ }^{206} \mathrm{Po}$ along the column using standard $\gamma$-spectrometry with lead collimator as described in [4].

When the resulting thermochromatograms were characterized by a sharp peak (with width estimated for the experimental conditions used in this work of 3 to maximum $5 \mathrm{~cm}$ ), they were simulated by a Monte Carlo code for gas chromatography [14] to determine the adsorption enthalpy of the adsorbed species.

The uncertainty of the calculated value of adsorption enthalpy, resulting from the uncertainty of the deposition temperature measurement, $\pm 30 \mathrm{~K}$, was estimated to be $\pm 6 \mathrm{~kJ} \mathrm{~mol}^{-1}$.

\section{Results and discussion}

The interaction of polonium with SS316L was investigated using different carrier gases to reproduce different conditions of MYRRHA, such as normal operational, maintenance and accidental scenarios. During normal operation conditions the cover gas will be inert or even slightly reducing. Thus, the first set of experiments was conducted using either inert or reducing carrier gas, namely dry helium and dry hydrogen. During ordinary maintenance ingress of oxygen is expected while in an accidental scenario, ingress of both oxygen and water is likely. The effects of oxygen and water on the polonium interaction with SS316L were investigated using either dry oxygen or moist carrier gas.
Table 1 summarizes the experimental conditions and the results of the performed investigations, in terms of deposition temperature, adsorption enthalpy and chemical speciation.

\subsection{Thermochromatography studies with helium as carrier gas}

Figure 2 shows the thermochromatograms resulting from the Exp. I and II, panel a and b, respectively. In both case the columns were not pretreated in hydrogen, and they were kept in the gradient furnace for $240 \mathrm{~min}$ in dry and moist helium, respectively, before starting the experiment. The water contents of the carrier gas of Exp. I ranged from 111 ppmv, at the beginning of the experiment, to $53.5 \mathrm{ppmv}$, at the end of the experiment. The contents of water in Exp. II approached the saturation value at room temperature (about 2 vol.\%). The obtained thermochromatograms, characterized by a sharp peak centered at $585 \mathrm{~K}$ and $556 \mathrm{~K}$, respectively, were simulated with the Monte Carlo code [14]. Best agreement between experiment and simulation was achieved with adsorption enthalpy values of $-147 \pm 6 \mathrm{~kJ} \mathrm{~mol}^{-1}$ and $-140 \pm 6 \mathrm{~kJ} \mathrm{~mol}^{-1}$, respectively.

Exp. I (a) and (b), Table 1, were also performed using dry helium as carrier gas, but here, the used columns were pretreated in hydrogen as described above and were kept in the gradient furnace for longer time, 300 and $840 \mathrm{~min}$, respectively, before the experiments. The obtained thermochromatograms were characterized by deposition temperatures of $583 \mathrm{~K}$ and $588 \mathrm{~K}$, respectively, and an adsorption enthalpy of $-146 \pm 6 \mathrm{~kJ} \mathrm{~mol}^{-1}$ (Table 1). In Exp. II (a) and (b), where moist helium was used as carrier gas, the columns were pretreated and kept in the gradient furnace for 270 and $840 \mathrm{~min}$, respectively, prior to the experiments. The resulting adsorption enthalpies were $-142 \pm 6 \mathrm{~kJ} \mathrm{~mol}^{-1}$ and $-135 \pm 6 \mathrm{~kJ} \mathrm{~mol}^{-1}$, respectively (Table 1). The values of adsorption enthalpy of the thermochromatography experiments performed using dry or moist helium range from $-147 \pm 6 \mathrm{~kJ} \mathrm{~mol}^{-1}$ to $-135 \pm 6 \mathrm{~kJ} \mathrm{~mol}^{-1}$. Considering the uncertainties of the calculated adsorptions enthalpies, $\pm 6 \mathrm{~kJ} \mathrm{~mol}^{-1}$, it is possible to conclude that the same polonium species was deposited in these experiments, independently of the pretreatment of the columns, moisture content and the time that the column was kept in the gradient furnace.

The polonium concentration in the experiments is so low that the collision between two atoms of polonium is improbable [15]. This excludes the formation of $\mathrm{Po}_{2}$. Based on the same reasoning the formation of BiPo, that 
Table 1: Experimental conditions and results of thermochromatography experiments.

\begin{tabular}{|c|c|c|c|c|c|c|c|}
\hline $\begin{array}{l}\text { Sample } \\
\text { number }\end{array}$ & Carrier gas & $\begin{array}{l}\text { Pretreatment } \\
\text { in hydrogen } \\
\text { gas }\end{array}$ & $\begin{array}{r}\text { Heating time of } \\
\text { column in the } \\
\text { gradient before } \\
\text { experiment/min }\end{array}$ & $\begin{array}{r}\text { Moisture content } \\
\text { of carrier gas/ } \\
\text { ppmv }^{\mathrm{a}}\end{array}$ & $\begin{array}{r}T_{\text {dep }} / \mathrm{K} \\
\text { uncertainty }= \pm 30 \mathrm{~K}\end{array}$ & $\begin{array}{r}\Delta H_{\text {ads }} / \mathrm{kJ} \mathrm{mol}^{-1} \\
\text { uncertainty }= \pm 6 \mathrm{~kJ} \mathrm{~mol}^{-1}\end{array}$ & $\begin{array}{l}\text { Chemical } \\
\text { species }\end{array}$ \\
\hline Exp. I & He dry & No & 240 & $111-53.5$ & 585 & -147 & Po \\
\hline Exp. I (a) & He dry & Yes & 300 & $111-77$ & 583 & -146 & Po \\
\hline Exp. I (b) & He dry & Yes & 840 & $77-26.5$ & 588 & -146 & Po \\
\hline Exp. II & He moist & No & 240 & $\sim 20,000$ & 556 & -140 & Po \\
\hline Exp. II (a) & He moist & Yes & 270 & $\sim 20,000$ & 587 & -142 & Po \\
\hline Exp. II (b) & He moist & Yes & 840 & $\sim 20,000$ & 546 & $-135^{c}$ & Po \\
\hline Exp. III & $\mathrm{H}_{2}$ dry & No & $840^{d}$ & $186-164$ & 547 & -136 & Po \\
\hline Exp. III (a) & $\mathrm{H}_{2}$ dry & No & 840 & $59.3-55.4$ & 815 & - & Po \\
\hline Exp. IV & $\mathrm{H}_{2}$ moist & No & 240 & $\sim 20,000$ & 606 & -148 & Po \\
\hline Exp. IV (a) & $\mathrm{H}_{2}$ moist & Yes & 300 & $\sim 20,000$ & 586 & -147 & Po \\
\hline Exp. V & $\mathrm{O}_{2}$ dry & No & 240 & $88.9-45.5$ & 896 & -225 & $\mathrm{PoO}_{2}$ \\
\hline Exp. V (a) & $\mathrm{O}_{2}$ dry & Yes & 240 & $71-49$ & 896 & -225 & $\mathrm{PoO}_{2}$ \\
\hline Exp. V (b) & $\mathrm{O}_{2}$ dry & Yes & 720 & $29-36.3$ & 870 & - & $\mathrm{PoO}_{2}$ \\
\hline Exp. V (c) & $\mathrm{O}_{2}$ dry & Yes & 1005 & $40-8.4$ & 808 & - & $\mathrm{PoO}_{2}$ \\
\hline Exp. V (d) & $\mathrm{O}_{2}$ dry & No & 1020 & $15.5-10.5$ & 798 & - & $\mathrm{PoO}_{2}$ \\
\hline Exp. VI & $\mathrm{O}_{2}$ moist & No & 210 & $\sim 20,000$ & 883 & -221 & $\mathrm{PoO}_{2}$ \\
\hline Exp. VI (a) & $\mathrm{O}_{2}$ moist & Yes & 210 & $\sim 20,000$ & 917 & -229 & $\mathrm{PoO}_{2}$ \\
\hline
\end{tabular}

${ }^{\mathrm{a}}$ For dried gases values measured at the beginning and at the end of the experiment are given. ${ }^{\mathrm{b}}$ Experiment performed for $1 \mathrm{~h}$. 'Broad deposition pattern. In helium atmosphere.

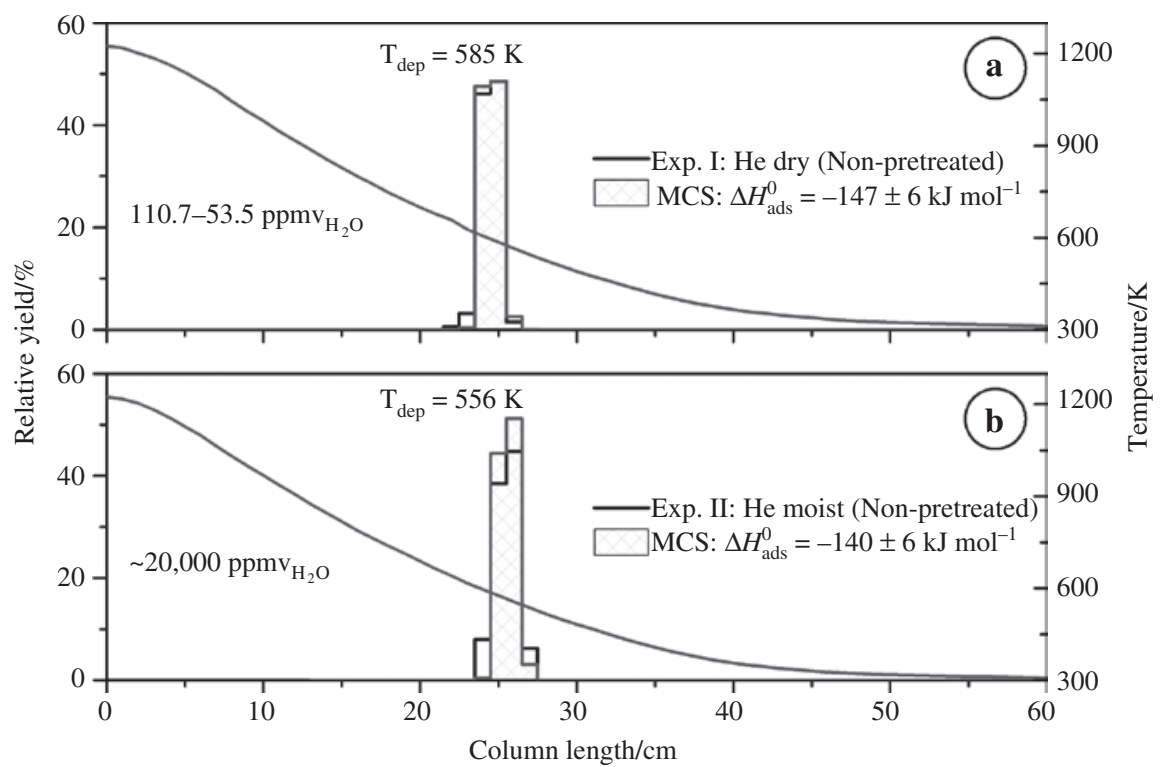

Figure 2: Thermochromatograms of ${ }^{206} \mathrm{Po}$ (black bars) using dry helium (Exp. I, panel a) and moist helium (Exp. II, panel b), respectively. The cross-hatched bars represent the corresponding Monte Carlo simulations.

could result by the reaction of polonium with ${ }^{206} \mathrm{Bi}$, which is always to some extent present in the starting materials as decay product of ${ }^{206} \mathrm{Po}$, could be excluded as well. The activity of ${ }^{206} \mathrm{Bi}$ was monitored in all experiments too and confirmed this assumption, as it was found to always deposit at higher temperatures than polonium. Since the use of inert carrier gas preserves the chemical state of polonium avoiding the formation of molecular species such as hydroxides, oxides or oxy-hydroxides, the depositions observed in Exp. I and II were tentatively assigned to monoatomic polonium. It is noteworthy that the presence of few volume percent of water in the carrier gas of Exp. II 
did not change the chemical speciation of polonium. This observation is in strong contrast to the behaviour of polonium in previous experiments performed using fused silica as stationary phase in similar conditions [4], where the formation of highly volatile polonium species in moist helium was observed.

The stationary phase, SS316L, contains iron, between $16-18 \%$ of chromium and $10-14 \%$ of nickel [17]. In ambient air and at room temperature, the surface of SS316L is covered by a thin layer of mixed iron and chromium oxides. The ratio of iron and chromium oxide to metals (iron oxide $/ \mathrm{Fe}^{0}$ and chromium oxide $/ \mathrm{Cr}^{0}$ ), which determines the chemical characteristics of the SS316L surface, depends on the chemical and/or mechanical processing of the surface [18]. Kerber and Tverberg [18] used XPS to study the variation of the surface chemistry of SS316L passivated with nitric acid for different times. They reported that the non-treated SS316L surface was characterized by the following ratios: $\mathrm{Cr}^{0} / \mathrm{Fe}^{0}=0.28$, $\mathrm{Fe}_{2} \mathrm{O}_{3} / \mathrm{Fe}^{0}=1$ and $\mathrm{Cr}_{2} \mathrm{O}_{3} / \mathrm{Cr}^{0}=3.2$. Therefore, we conclude that the calculated adsorption enthalpy is related to the interactions of polonium with a surface containing iron-chromium mixed oxide and some metallic Fe and/ or $\mathrm{Cr}$ sites. Thus, the slightly more negative value determined for the adsorption enthalpy on SS316L compared to the one of polonium on fused silica $\left(-125 \pm 5 \mathrm{~kJ} \mathrm{~mol}^{-1}\right.$ [4]) could be caused by the increased metallic character of the surface. This will be further discussed below in context with the influence of hydrogen on the adsorption behaviour.

\subsection{Thermochromatography studies with hydrogen as carrier gas}

Dry hydrogen was used as carrier gas in Exp. III. In this case, a non-pretreated column was used and the temperature gradient was established within $840 \mathrm{~min}$ in helium atmosphere. Immediately before starting the experiment the carrier gas was switched to dry hydrogen. The exposure of the non-reduced column surface to hydrogen caused a comparatively high water content in the gas phase throughout the experiment, ranging from 186 ppmv to 164 ppmv. The obtained thermochromatogram, Figure 3 panel a, reveals a single sharp peak centred at $547 \mathrm{~K}$ and a calculated adsorption enthalpy of $-136 \pm 6 \mathrm{~kJ} \mathrm{~mol}^{-1}$. This value is in the range of adsorption enthalpies calculated for the experiment performed using dry or moist helium, indicating that also in this case the deposited species is monoatomic polonium. Figure 3 panel $b$ shows the thermochromatogram of Exp. III (a) obtained in dry hydrogen but, differently from Exp. III, it was used a pretreated column which was kept in the gradient furnace for $840 \mathrm{~min}$ in hydrogen atmosphere. The result indicates a broadening of the peak and a shift of the deposition to significantly higher temperature, which could be caused by two different effects: a modification of the chemical speciation of polonium or a modification of the stationary phase. The first hypothesis can be excluded since the only species that can be formed at this temperature and under these reducing conditions is elemental polonium. In fact, polonium

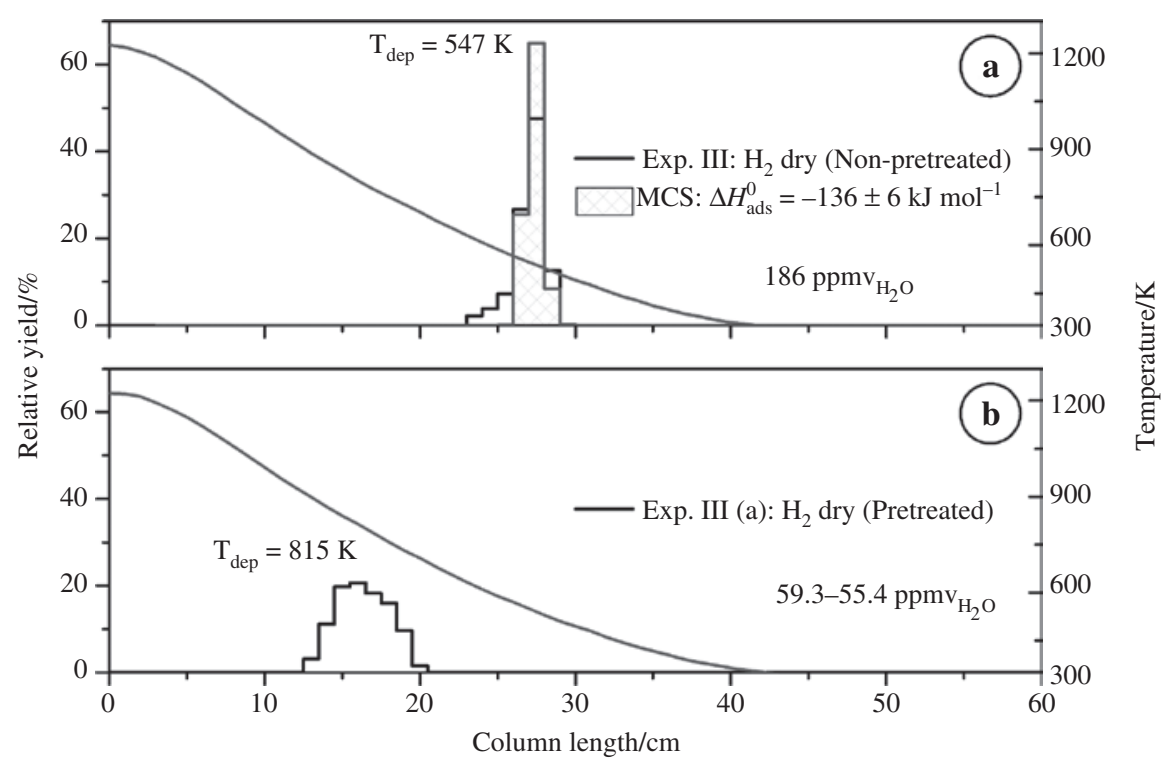

Figure 3: Thermochromatograms of ${ }^{206} \mathrm{Po}$ (black bars) using dry hydrogen in non-pretreated (Exp. III, panel a) and pretreated (Exp. III (a), panel b) columns, respectively. The cross-hatched bars represent the relative Monte Carlo simulations. 


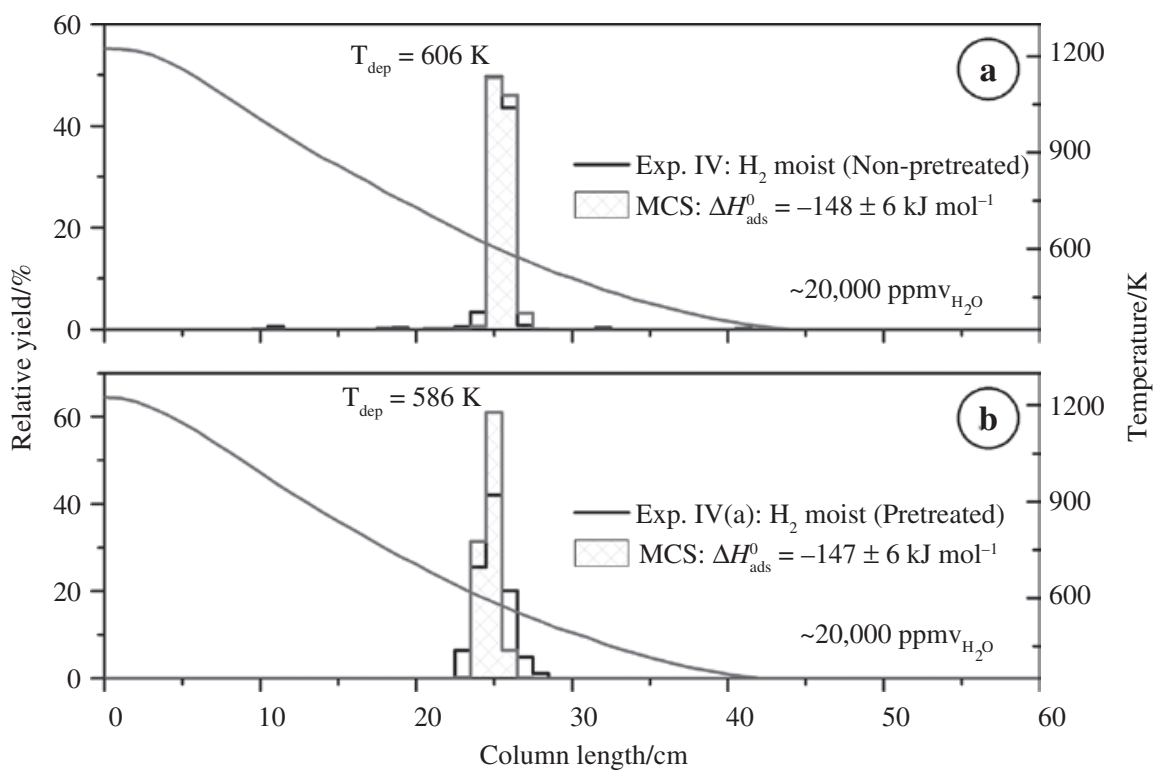

Figure 4: Thermochromatograms of ${ }^{206} \mathrm{Po}$ using moist hydrogen. The SS316 L column was not pretreated in Exp. IV, panel a, while it was pretreated and kept $14 \mathrm{~h}$ in the gradient furnace under $\mathrm{H}_{2}$ before the experiment in Exp. IV (a), panel b.

hydride, if formed, would decompose above $273 \mathrm{~K}$ [19], while the formation of oxides or oxy-hydroxides species is unlikely due to the strong reducing potential of the carrier gas. Thus, the argument of the column surface modification must be considered. Indeed, hydrogen, used as carrier gas, can modify the SS316L surface, see e. g. Ref. [20]. Considering that the column of Exp. III (a) was kept in the gradient furnace in hydrogen atmosphere for $14 \mathrm{~h}$ before the starting of the experiment, partial reduction of the surface to a metallic one, with a consequent decrease of the ratio of iron and chromium oxide to metals on the surface can occur. This translates into an increase of the metallic character of the stationary phase. The adsorption enthalpy of polonium on metallic iron and on metallic chromium was predicted using a semi-empirical model described in [21], as $-346 \mathrm{~kJ} \mathrm{~mol}^{-1}$ and $-409 \mathrm{~kJ} \mathrm{~mol}^{-1}$, respectively, while on nickel, also present in the SS316L surface [18], was calculated to be $-220 \mathrm{~kJ} \mathrm{~mol}^{-1}$. This indicates that polonium has a stronger affinity for the metals compared to the oxides, supporting the conclusion that during Exp. III (a) the surface of the stationary phase was partially reduced. In additions, the broadening of the thermochromatograms of Exp. III (a) reveals a larger chemical heterogeneity of the surface, i. e. mixing of both oxides (with different ratio of iron oxide and chromium oxide) and metals (with different ratio iron/chromium).

This phenomenon was not observed in Exp. IV and Exp. IV (a), Figure 4, where moist hydrogen $(\sim 2$ vol.\% of water) was used as carrier gas. The obtained thermochromatograms, in fact, outlines a single sharp peak centred at $606 \mathrm{~K}$ and $586 \mathrm{~K}$, respectively, resulting in an adsorption enthalpy of $-148 \pm 6 \mathrm{~kJ} \mathrm{~mol}^{-1}$ and $-147 \pm 6 \mathrm{~kJ} \mathrm{~mol}^{-1}$, respectively. These values, considering the experimental uncertainty, are the same as the one assigned to the interaction of monoatomic polonium with a surface containing metal oxides with a small fraction of metals. This indicates that the presence of water in the gas phase has prevented the partial reduction of the stationary phase, keeping the ratio of iron and chromium oxide to metal similar to the one of the initial conditions. It is important to notice that the presence of water did not change the chemical speciation of polonium since the deposited species is considered to be monoatomic polonium, similarly to what was previously observed e.g. in Exp. I and Exp. II. This is again in contrast to similar experiments performed in fused silica tubes, where the formation of polonium species more volatile than monoatomic polonium was observed in moist hydrogen carrier gas.

\subsection{Thermochromatography studies with oxygen as carrier gas}

Exp. V was performed using dry oxygen (water contents between 88.9 and $45.5 \mathrm{ppmv}$ ) and a not pretreated column. The obtained thermochromatogram, centred at $896 \mathrm{~K}$, was best reproduced by Monte-Carlo simulations using adsorption enthalpies of $-225 \pm 6 \mathrm{~kJ} \mathrm{~mol}^{-1}$, Figure 5, panel a. The sharpness of the peaks indicates the adsorption of 
one single polonium species, which can be identified as polonium dioxide, since +4 is the most stable valence state of polonium in pure oxygen [22]. The deduced adsorption enthalpies agree well with those found for polonium dioxide on fused silica, $-215 \pm 6 \mathrm{~kJ} \mathrm{~mol}^{-1}$ [4]. Repetition of the experiment at same conditions, using a pretreated column, Figure 5, panel b, led to the same results in terms of deposition temperature and adsorption enthalpy, indicating that the pretreatment does not affect the resulting adsorption/desorption equilibrium, most likely because the column surface that had been reduced in the pretreatment was oxidized again when the column was exposed to oxygen for $240 \mathrm{~min}$ in the temperature gradient.

The value of adsorption enthalpies calculated for Exp. $V$ and Exp. $V($ a) refers to the interaction of polonium dioxide with a SS316L surface which was exposed to pure oxygen for $240 \mathrm{~min}$ before the starting of the experiment. In the light of the results described above, which revealed an influence of prolonged exposure of the column to hydrogen on the adsorption behaviour, it was decided to investigate the effects of the oxygen exposure on the column surface by doing a series of experiments where the stationary phases were kept under dry oxygen before the thermochromatography experiment for intervals ranging from $240 \mathrm{~min}$ up to $1020 \mathrm{~min}$. The effects of the use of pretreated and non-treated columns were evaluated as well. Figure 5, panels c, d and e, illustrate the thermochromatograms of this series of experiments. In particular, Exp. V (b), Exp. V (c) were performed using pretreated columns which were kept in the gradient furnace, in dry oxygen atmosphere, before the experiment for 720 and 1005 min, respectively. Exp. V (d) was done using a nontreated column kept in the gradient furnace for $1020 \mathrm{~min}$. From the resulting thermochromatograms it is possible to see that the deposition pattern broaden with the oxidation time, while the deposition temperatures decrease. The broadening of the patterns, as stated before, could be again induced by two main processes: 1) The modification of the chemical state of the adsorbed species; and/ or 2) The modification of the stationary phase surface. The dryness and the strong oxidizing potential of the used carrier gas excludes, on first approximation, the formation of species other than polonium dioxide, such as oxyhydroxides, with different adsorption enthalpies. This indicates that an increase of the chemical heterogeneity of the column surface is responsible for the broadening. On the other hand, the decrease of the deposition temperature suggests that the affinity of polonium oxide for the stationary phase decreases with increasing surface oxidation. No effects could be attributed to the pretreatment of the column of Exp. V (d).

Exp. VI and Exp. VI (a), conducted using moist oxygen ( $\sim 2$ vol.\% of water), are presented in Figure 6, panel a and $\mathrm{b}$, respectively. The thermochromatograms are centered at $883 \mathrm{~K}$ and $917 \mathrm{~K}$, respectively, and the adsorption enthalpies were calculated as $-221 \pm 6 \mathrm{~kJ} \mathrm{~mol}^{-1}$, and $-229 \pm 6 \mathrm{~kJ} \mathrm{~mol}^{-1}$, respectively. Considering the uncertainties of the calculated adsorption enthalpies, the species

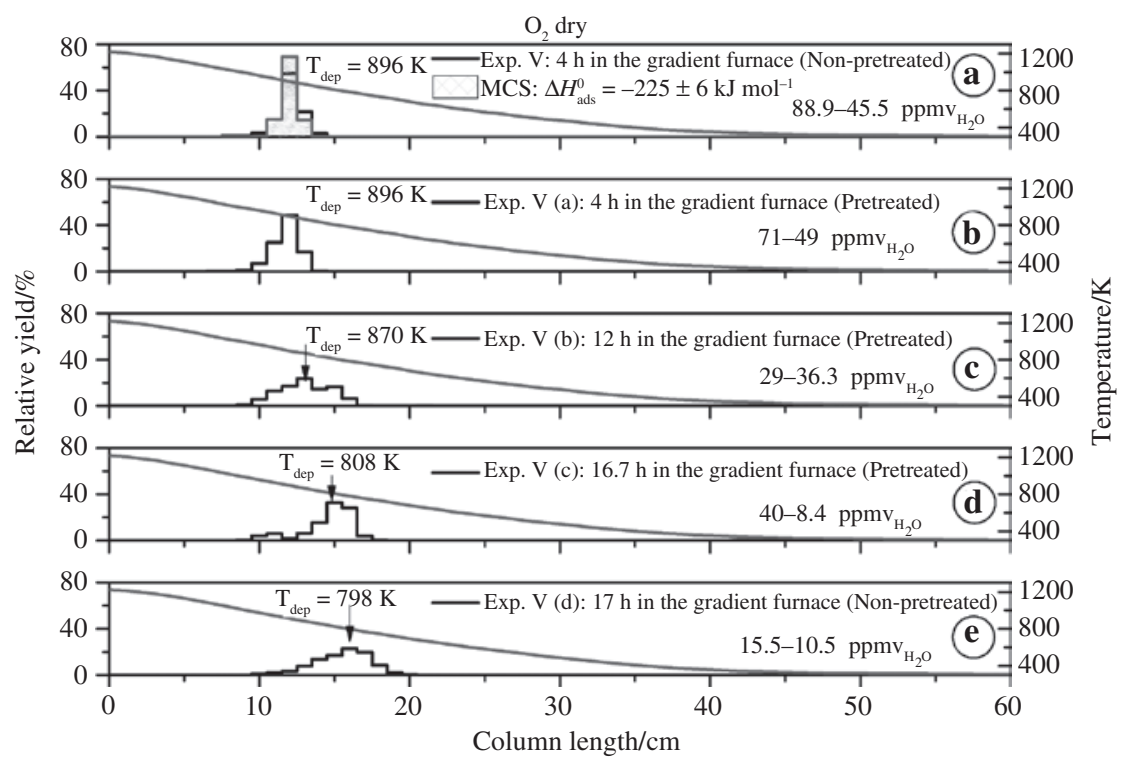

Figure 5: Thermochromatograms of ${ }^{206} \mathrm{Po}$ using dry oxygen as carrier gas. The experiments differ in terms of time the columns were kept in the gradient furnace before the corresponding experiment. Panel (a) Exp. V and panel (b) Exp. V (a): 4 h. Panel (c) Exp. V (b): 12 h. Panel (d) Exp. V (c): 16.7 h. Panel (e) Exp. V (d): 17 h. 


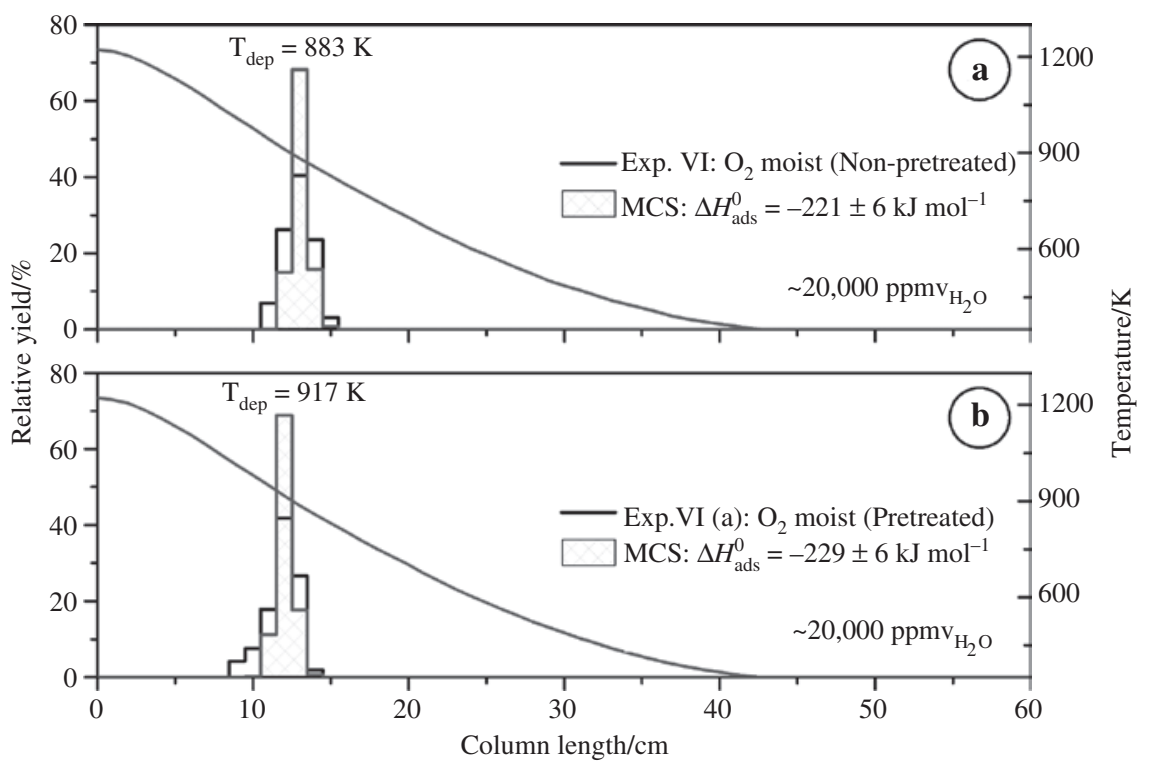

Figure 6: Thermochromatograms of ${ }^{206}$ Po using moist oxygen as carrier gas. In Exp. VI, panel (a), a non-pretreated column was used, while in Exp. VI (a), panel (b), a pretreated column was used.

deposited in both experiments is considered to be the same as in Exp. V, i. e. polonium dioxide. The result of Exp. VI and Exp. VI (a) indicates that no chemical reactions between polonium dioxide and water was superimposing the adsorption/desorption equilibrium. This reaction could induce the formation of different species, such as oxyhydroxides, with lower affinity for the oxidized stationary phase, as reported in [4]. In other words, the presence of water in the gas phase did not induce transport processes.

\subsection{Polonium not deposited on the column}

A silver tube, $2 \mathrm{~cm}$ length and $0.020 \pm 0.003 \mathrm{~mm}$ thickness, was lining the inner surface at end of each column downstream from the gradient to catch ${ }^{206}$ Po not retained in the column. The adsorption enthalpy of polonium on silver was determined as $-216 \mathrm{~kJ} \mathrm{~mol}^{-1}$ and $-230 \mathrm{~kJ} \mathrm{~mol}^{-1}$ in inert and reducing atmosphere, respectively, and as $-234 \mathrm{~kJ} \mathrm{~mol}^{-1}$ in oxidizing conditions [10]. These values, if compared with the value of adsorption enthalpy reported in this work, indicate a stronger affinity of polonium for silver than for SS316L. Thus, if part of the polonium transported in the column was not adsorbed into the SS316L surface during its way along the temperature gradient, it would be partially or totally trapped onto the silver surface, thus enabling us to evaluate if all the polonium desorbed from the starting material remains adsorbed on the SS316L column.
The overall measurement results are summarized in Table 2 in terms of fraction of the total polonium retrieved in the silver tubes after the experiments. Between 5 and $232 \mathrm{ppm}$ of the polonium evaporated from the starting sample were transported through the SS316L column and adsorbed on the silver tubes, meaning that a small but not negligible fraction of polonium was not retained on the SS316L surface. No correlation between the amount of

Table 2: Fractions of polonium measured on the silver tubes.

\begin{tabular}{llrrr}
\hline $\begin{array}{l}\text { Sample } \\
\text { number }\end{array}$ & Carrier & $\begin{array}{r}\text { Fraction } \\
\text { of Po on } \\
\text { Ag/ppm }\end{array}$ & $\begin{array}{r}\text { Po on Ag } \\
\text { upstream/ } \\
\text { ppm }\end{array}$ & $\begin{array}{r}\text { Po on Ag } \\
\text { downstream/ } \\
\text { ppm }\end{array}$ \\
\hline Exp. I & He dry & 79 & 12 & 67 \\
Exp. I (a) & He dry & 131 & 20 & 111 \\
Exp. I (b) & He dry & 279 & 30 & 249 \\
Exp. II & He moist & 18 & 18 & 0 \\
Exp. II (a) & He moist & 116 & 0 & 116 \\
Exp. II (b) & He moist & 167 & 29 & 138 \\
Exp. III & $\mathrm{H}_{2}$ dry & 57 & 144 & 201 \\
Exp. III (a) & $\mathrm{H}_{2}$ dry & 164 & 9 & 156 \\
Exp. IV & $\mathrm{H}_{2}$ moist & 69 & 49 & 20 \\
Exp. IV (a) & $\mathrm{H}_{2}$ moist & 40 & 40 & 0 \\
Exp. V & $\mathrm{O}_{2}$ dry & 5 & 5 & 0 \\
Exp. V (a) & $\mathrm{O}_{2}$ dry & 216 & 12 & 204 \\
Exp. V (b) & $\mathrm{O}_{2}$ dry & 10 & 3 & 7 \\
Exp. V (c) & $\mathrm{O}_{2}$ dry & 63 & 33 & 30 \\
Exp. V (d) & $\mathrm{O}_{2}$ dry & 174 & 70 & 104 \\
Exp. VI & $\mathrm{O}_{2}$ moist & 232 & 68 & 164 \\
Exp. VI (a) & $\mathrm{O}_{2}$ moist & 6 & 14 & 20 \\
\hline
\end{tabular}


polonium measured on the silver tubes and experimental conditions was found. For example, the fractions of polonium measured on the silver tubes of the five experiments performed using dry oxygen are not consistent.

All silver tubes were cut in two equivalent parts in order to evaluate if all the non-retained polonium was trapped on the silver surface. Thus, the two parts, upstream and downstream (the last portion was the one closer to the outlet of the thermochromatography set-up), were measured separately. The results of these measurements are summarized too in Table 2. Also in this case no correlation between the results and experimental conditions was found. In some measurements, e. g. Exp. VI, the fraction of polonium measured in the downstream part is two times larger as that of the upstream part. In some other experiments, e. g. Exp. III and V, the fraction of polonium measured on the downstream part of the silver is 17 times larger than the one on the upstream part. There are also a few experiments where more polonium deposited on the upstream part than on the downstream part. This indicates that it is not possible to state that all the polonium not retained in the SS316L column was trapped on the silver foils. Thus, the reported values must be considered as indicative and, in any case, as lower limits.

\section{Conclusions}

The adsorption enthalpy of polonium on SS316L in inert gas was measured as $-147 \pm 6 \mathrm{~kJ} \mathrm{~mol}^{-1}$. This value is slightly more negative than the adsorption enthalpy of polonium on silicon dioxide in form of fused silica, indicating that either the SS316L was not fully oxidized, or the affinity of polonium for the oxide layer on 316L steel is slightly higher than that for fused silica. The use of reducing carrier gas, hydrogen, did not induce modifications of the chemical speciation of polonium and stabilized its elemental atomic state. On the other hand, hydrogen induced chemical modifications of the SS316L surface, increasing its metallic character and thus enhancing the affinity of polonium to SS316L. Thus, a potentially slightly reducing atmosphere of the MYRRHA cover gas, could facilitate the adsorption of polonium on the structural material reducing the fraction of this hazardous element in the gas phase. The presence of water in the carrier gas, up to $\sim 2$ vol.\%, at reducing and inert conditions did not induce variation of the chemical state of polonium, although prolonged exposures of the SS316L to water could increase the iron and chromium oxide fraction on the surface and thus the decrease of polonium affinity. Oxidizing gas induces the formation of polonium dioxide, which has a higher adsorption enthalpy on SS316L, $-225 \pm 6 \mathrm{~kJ} \mathrm{~mol}^{-1}$. Also in this case, prolonged exposures of the SS316L to oxygen increase the oxide fraction on the surface which causes a weakening of the adsorption interaction. Based on these results, it can be concluded that accidental ingress of oxygen into MYRRHA cover gas will not cause a volatilization of micro amounts of polonium eventually adsorbed on the SS316L surface. No effects of water on the chemical speciation of both the SS316L surface and the polonium oxide were evident from the results obtained using moist oxygen as carrier gas.

Further study of certain aspects of polonium volatilization is still required. In particular, more experiments will be needed to verify whether higher amount or longer exposure time of water may induce transport reactions with formation of different polonium species with less affinity to the SS316L surface. Moreover, the interaction of bismuth and SS316L must be addressed, since this element is present in the cover gas in much higher concentrations than polonium. Macroscopic amounts of bismuth species adsorbed on the S316L surface might change the interaction with polonium as well as macroscopic amounts of polonium.

It was found that more than $5 \mathrm{ppm}$ of polonium were not deposited onto the SS316L column surface during all the performed thermochromatography experiments.

This indicates that even though polonium interacts relatively strongly with the structural material, a small fraction of it may stay in the gas phase. The nature of this polonium fraction remains to be clarified.

Acknowledgments: This work was supported by the project MYRTE under EURATOM HORIZON2020 Grant Agreement No. 662186.

\section{References}

1. Rubbia, C.: A high gain energy amplifier operated with fast neutrons. AIP Conf. Proc. 346, 44 (1995).

2. Salvatores, M., Palmiotti, G.: Radioactive waste partitioning and transmutation within advanced fuel cycles: achievements and challenges. Prog. Part. Nucl. Phys. 66, 144 (2011).

3. Aït Abderrahim, H., Baeten, P., De Bruyn, D., Fernandez, R.: MYRRHA - A multi-purpose fast spectrum research reactor. Energy Convers. Manage. 63, 4 (2012).

4. Maugeri, E. A., Neuhausen, J., Eichler, R., Piguet, D., Mendonça, T. M., Stora, T., Schumann, D.: Thermochromatography study of volatile polonium species in various gas atmospheres. J. Nucl. Mater. 450, 292 (2014).

5. Buongiorno, J., Loewen, E. P., Czerwinski, K., Larson, C.: Studies of polonium removal from molten lead-bismuth for lead-alloycooled reactor applications. Nucl. Technol. 147, 406 (2004). 
6. Pankratov, D. V., Efimov, E. I., Toshinskii, G. I., Ryabaya, L. D.: Analysis of the polonium hazard in nuclear power systems with lead-bismuth coolant. At. Energ. 97, 559 (2004).

7. Heinitz, S.: Investigations on physico-chemical aspects of leadbased alloys for nuclear applications, PhD Thesis, University of Bern, 2013.

8. Heinitz, S., Neuhausen, J., Schumann, D.: Alkaline extraction of polonium from liquid lead bismuth eutectic. J. Nucl. Mater. 414, 221 (2011)

9. Maugeri, E. A., Neuhausen, J., Eichler, R., Dressler, R., Rijpstra, K., Cottenier, S., Piguet, D., Vogele, A., Schumann, D.: Adsorption of volatile polonium and bismuth species on metals in various gas atmospheres: Part I - Adsorption of volatile polonium and bismuth on gold. Radiochim. Acta 104, 757 (2016).

10. Maugeri, E. A., Neuhausen, J., Misiak, R., Eichler, R., Dressler, R., Piguet, D., Vogele, A., Schumann, D.: Adsorption of volatile polonium species on metals in various gas atmospheres: Part II - Adsorption of volatile polonium on platinum, silver and palladium. Radiochim. Acta 104, 769 (2016).

11. Obara, T., Koga, T., Miura, T., Sekimoto, H.: Polonium evaporation and adhesion experiments for the development of polonium filter in lead-bismuth cooled reactors. Prog. Nucl. Energ. 50, 556 (2008).

12. Obara, T., Miura, T., Sekimoto, H.: Fundamental study of polonium contamination by neutron irradiated lead-bismuth eutectic. J. Nucl. Mater. 343, 297 (2005).
13. Obara, T., Yamazawa, Y., Sasa, T.: Polonium decontamination performance of stainless steel mesh filter for lead alloy-cooled reactors. Prog. Nucl. Energ. 53, 1056 (2011).

14. Zvara, I.: Simulation of thermochromatographic processes by the Monte Carlo method. Radiochim. Acta 38, 95 (1985).

15. Zvara, I.: The Inorganic Radiochemistry of Heavy Elements. Springer, Netherlands (2010).

16. Neuhausen, J., Köster, U., Eichler, B.: Investigation of evaporation characteristics of polonium and its lighter homologues selenium and tellurium from liquid $\mathrm{Pb}$-Bi-eutecticum. Radiochim. Acta 92, 917 (2004).

17. Oberg, E., Press, I.: Machinery's Handbook 29th Edition - Full Book. Industrial Press, New York (2012).

18. Kerber, S. J.,Tverberg, J.: Stainless steel surface analysis. Adv. Mater. Processes 158, 33 (2000)

19. Greenwood, N. N., Earnshaw, A.: Chemistry of the Elements. Elsevier Science \& Technology Books, Pergamon Press, Oxford (Oxfordshire), New York (1984).

20. Hara, S., Abe, T., Enoeda, M., Takatsu, H.: Effects of hydrogen atmosphere on mechanical properties and surface conditions of a reduced activation ferritic steel F82H. J. Nucl. Mater. 258-263, Part 2, 1280 (1998)

21. Eichler, B., Rossbach, H.: Adsorption of volatile metals on metalsurfaces and its application in nuclear chemistry. 1. Calculation of adsorption enthalpies for hypothetical superheavy elements with Z around 114. Radiochim. Acta 33, 121 (1983).

22. Abakumov, A. S.: Thermal reactions of polonium. Russ. Chem. Rev. 51, 622 (1982). 